\title{
A lattice gas of prime numbers and the Riemann Hypothesis
}

\author{
Fernando Vericat \\ Grupo de Aplicaciones Matemáticas y Estadísticas de la Facultad de \\ Ingeniería (GAMEFI). Universidad Nacional de La Plata, Argentind*
}

\begin{abstract}
In recent years, there has been some interest in applying ideas and methods taken from Physics in order to approach several challenging mathematical problems, particularly the Riemann Hypothesis. Most of these kind of contributions are suggested by some quantum statistical physics problems or by questions originated in chaos theory. In this article we show that the real part of the non-trivial zeros of the Riemann zeta function extremizes the grand potential corresponding to a simple model of one-dimensional classical lattice gas, the critical point being located at $1 / 2$ as the Riemann Hypothesis claims.

PACS numbers: 02.10.De, 05.20.Gg, 05.50.+q
\end{abstract}

Keywords: Lattice gas, prime numbers, variational principle, Riemann Hypothesis

\footnotetext{
*Also at Instituto de Física de Líquidos y Sistemas Biológicos (IFLYSIB)-CONICET-CCT La Plata, Argentina.; E-mail: vericat@iflysib.unlp.edu.ar
} 


\section{INTRODUCTION}

The relation between physics and number theory has a long and fruitful history as the two books referenced [1] and [2] suggest. This link is in both directions: from number theory to physics and from physics to number theory. At the beginning, the first direction was the more frequently transited and so many number theoretic functions (and concepts in general) were used in field theory, in classical and quantum statistical physics and also in chaos theory. In this sense the Riemann zeta function[3] defined by $\zeta(s):=\sum_{n=1}^{\infty} n^{-s}$ in the half-plane $\Re(s)>1$ and by analytical continuation in the whole complex plane, plays an important role.

Given the ubiquitousness of $\zeta(s)$ in physics as well as in mathematics itself, its behavior, particularly the properties of its zeros, has been much studied. Nowadays we know that $\zeta(s)=0$ has as trivial solutions the negative integers $-2 k$ with $k \in \mathbb{N}$. The so-called nontrivial zeros are complex numbers most of whose properties are also known. In an application of $\zeta(s)$ to a number theoretic problem, specifically the distribution of the prime numbers, Riemann conjectured more than 150 years ago that all the complex zeros have 1/2 as real part[3]. This statement is the famous Riemann Hypothesis whose proof has unsuccessfully involved many mathematicians since then. This apparent inaccessibility to their proof from a full rigorous mathematical point of view has, perhaps, motived the interest in applying ideas and methods taken from physics in order to approach the Riemann Hypothesis in particular as well as several other number theoretic problems. In this way we can mention the work done around the idea of Hilbert and Polya, proposed at the beginning of the past century, that the complex zeros of $\zeta(s)$ constitute the spectrum of an operator $\mathcal{R}=1 / 2 \mathcal{I}+i \mathcal{H}$ where the Hamiltonian $\mathcal{H}$ is self-adjoint. Also, it is worth mentioning diverse applications of statistical physics such as the use of random walks in order to approach the Riemann Hypothesis [4], [5] or the search for statistical mechanics models whose partition functions be related to $\zeta(s)$ by using spin chains [6] or gases of harmonic oscillators [7]. These (and several others) contributions of physics to number theory can be seen in the excellent review by Schumayer and Hutchinson[8].

In this article, following the way from physics to number theory, we give support to the Riemann Hypothesis by extremizing the grand potential of a simple classical one-dimensional lattice gas of prime numbers. In next section we discuss the model and the equilibrium 
statistical mechanics functions that we need for our analysis, particularly the grand potential of the system. Section 3 is devoted to present the explicit formula given by Riemann for the prime-counting function in terms of the zeros of the zeta function and, as a natural consequence of the enunciation of the properties of these ones, to state again the Riemann Hypothesis. Finally in section 4 we give support to the Riemann Hypothesis from our model by showing that the real part of the complex zeros of the zeta function must extremize the grand potential obtained in section 2. Three appendices are considered in order to justify some equations of the main text.

\section{LATTICE GAS OF PRIME NUMBERS}

Physically our model is very simple: a one-dimensional lattice gas in the grand canonical ensemble. The lattice is an interval of the natural numbers: $[1, M] \subset \mathbb{N}$ where $M$ is large enough (eventually, in the thermodynamic limit, $M \rightarrow \infty$ ). The system is in contact with a particles reservoir characterized by the chemical potential $\mu$ and a heat reservoir at temperature $T$. For $N$ particles the configuration of the system is given by $\omega \equiv\left(\omega_{N}, N\right)$ where $\omega_{N} \equiv\left(i_{1}, i_{2}, \cdots, i_{N}\right)$. The coordinates $i_{\alpha}(\alpha=1,2, \cdots, N)$ take values in $[1, M]$ and $N$ ranges, in principle, between 0 and $\infty$. The set of such configurations, the configuration space, will be denoted $\Lambda$.

We assume that each site in the lattice can have at most one particle, so we are considering a hard point pair potential

$$
u_{2}\left(i_{\alpha}, i_{\beta}\right)= \begin{cases}\infty & \text { if } i_{\alpha}=i_{\beta} \\ 0 & \text { if } i_{\alpha} \neq i_{\beta}\end{cases}
$$

Also we assume that the particles are subject to a one-point potential such that they can just occupy sites in the lattice which are prime numbers:

$$
u_{1}\left(i_{\alpha}\right)=\left\{\begin{array}{l}
0 \text { if } i_{\alpha} \text { is prime } \\
\infty \text { if } i_{\alpha} \text { is composite }
\end{array}\right.
$$

By the way, an example of explicit function with this form is [9]:

$$
u_{1}\left(i_{\alpha}\right)=-\log \left[\sin ^{2}\left\{\pi\left[\left(i_{\alpha}-1\right) !\right]^{2} / i_{\alpha}\right\} / \sin ^{2}\left\{\pi / i_{\alpha}\right\}\right] .
$$

The energy for the configuration $\omega$ is $u(\omega)=\sum_{\alpha=1}^{N} u_{1}\left(i_{\alpha}\right)+\sum_{\alpha<\beta}^{N} u_{2}\left(i_{\alpha}, i_{\beta}\right)$. 
Mathematically, a state of the system is a probability vector $\nu=(\nu(\omega) \mid \omega \in \Lambda)$. The set of all states is denoted by $\mathcal{M}$. In the state $\nu$ the system has the mean energy $\nu(u):=\sum_{\omega \in \Lambda} \nu(\omega) u(\omega)$. The (grand) partition function of $u$ is defined $\Xi(\beta, \mu):=$ $\sum_{\omega \in \Lambda} \exp [-\beta(u(\omega)-\mu N)]$ where $\beta=\left(k_{B} T\right)^{-1}$ with $k_{B}$ the Boltzmann constant. For the parameters $\beta, M$ and $\mu$ the Gibbs measure is defined

$$
\nu_{0}(\omega):=\frac{1}{\Xi(\beta, M, \mu)} \exp [-\beta(u(\omega)-\mu N)] .
$$

Given a measure $\nu$ we have the entropy $H(\nu):=-\sum_{\omega \in \Lambda} \nu(\omega) \log \nu(\omega)$ and the grand potential

$$
\Omega[\nu]:=\nu(u)-\frac{1}{\beta} H(\nu)-\mu \nu(N)
$$

with $\nu(N):=\sum_{\omega \in \Lambda} \nu(\omega) N$.

To introduce the notion of equilibrium state we consider a variational principle for finite systems $(|\Lambda|=$ finite) according to which, for given energy $u$ and parameters $\beta, M$ and $\mu$, the Gibbs measure satisfies

$$
\Omega\left[\nu_{0}\right]=-\frac{1}{\beta} \log \Xi(\beta, M, \mu)=\inf _{\nu \in \mathcal{M}}\left(\nu(u)-\frac{1}{\beta} H(\nu)-\mu \nu(N)\right) .
$$

A measure that attains this infimum is called an equilibrium state. Gibbs measure $\nu_{0}$ is thus an equilibrium state. The result given by Eq.(5) is easily demonstrated[10] by using Jensen inequality applied to the concave function $x \mapsto \ln x$ (see Appendix A). The principle can be expressed saying that for any measure $\nu$ is $\Omega[\nu] \geqslant \Omega\left[\nu_{0}\right]=-\frac{1}{\beta} \log \Xi(\beta, M, \mu)$ with equality if and only if $\nu=\nu_{0}$.

Turning to our model, the grand potential for the Gibbs state is easily calculated if we explicitly write the grand partition function $(z=\exp [\beta \mu])$ :

$$
\Xi(\beta, M, \mu)=\sum_{N=0}^{\infty} \frac{z^{N}}{N !} \sum_{i_{1}=1}^{M} \sum_{i_{2}=1}^{M} \cdots \sum_{i_{N}=1}^{M} \exp \left[-\beta\left(\sum_{\alpha=1}^{N} u_{1}\left(i_{\alpha}\right)+\sum_{\alpha<\beta}^{N} u_{2}\left(i_{\alpha}, i_{\beta}\right)\right)\right]
$$

and observe that, because of the limitation of occupation to just the prime numbers (Eq22), the coordinates $i_{\alpha}(\alpha=1,2, \cdots, N)$ can take values only among the $\pi(M)$ prime numbers that exist in the interval $[1, M]$. Also, because of the impenetrability of the particles (Eq,1), the choice can be made of $\pi(M) ! /[\pi(M)-N]$ ! manners. We obtain 


$$
\Xi(\beta, M, \mu)=\sum_{N=0}^{\pi(M)}\left(\begin{array}{c}
\pi(M) \\
N
\end{array}\right) z^{N}=(1+z)^{\pi(M)}
$$

and

$$
\Omega\left[\nu_{0}\right]=-\frac{1}{\beta} \log (1+z)^{\pi(M)} .
$$

\section{RIEMANN HYPOTHESIS}

The prime-counting function $\pi(x)$ is the number of prime numbers less or equal than $x$. In his 1859 classic memoir to the Berlin Academy of Sciences (see for example the monograph by Edwards[3] for a translation), Riemann gave an explicit formula for $\pi(x)$ in terms of the zeros and the pole of the analytical continuation of $\zeta(s)[3]$ :

$$
\pi(x)=\bar{\pi}(x)+\widetilde{\pi}(x)
$$

where the smooth part, to which contribute the pole at $s=1$ and the integer zeros of $\zeta(s)$, is given by

$$
\bar{\pi}(x)=\sum_{n=1}^{\infty} \frac{\mu(n)}{n}\left[\operatorname{Li}\left(x^{1 / n}\right)-\sum_{k=1}^{\infty} \operatorname{Li}\left(x^{-2 k / n}\right)\right],
$$

with $\operatorname{Li}(x)$ the logarithmic integral function and $\mu(n)$ the Möbius function defined

$$
\mu(n)=\left\{\begin{array}{l}
0 \text { if } n \text { has one or more repeated prime factors } \\
(-1)^{r} \text { if } n \text { is a product of } r \text { distinct primes } \\
1 \text { if } n=1 .
\end{array}\right.
$$

The complex zeros, on the other hand, contribute to the oscillatory part

$$
\tilde{\pi}(x)=-2 \operatorname{Re} \sum_{n=1}^{\infty} \frac{\mu(n)}{n} \sum_{\alpha=1}^{\infty} \operatorname{Li}\left(x^{\left(\sigma_{\alpha}+i t_{\alpha}\right) / n}\right),
$$

where the real numbers $\sigma_{\alpha}$ and $t_{\alpha}$ are the real and imaginary part, respectively, of the complex number $\rho_{\alpha}=\sigma_{\alpha}+i t_{\alpha}$ that verifies $\zeta\left(\rho_{\alpha}\right)=0$. 
For $x$ large enough the non-oscillatory and oscillatory parts can be written, respectively, as

$$
\bar{\pi}(x) \approx \frac{x}{\log x}
$$

and (see Appendix B)

$$
\widetilde{\pi}(x) \approx-\frac{2}{\log x} \sum_{\alpha=1}^{\infty} \frac{x^{\sigma_{\alpha}}}{\left(\sigma_{\alpha}^{2}+t_{\alpha}^{2}\right)}\left[\sigma_{\alpha} \cos \left(t_{\alpha} \log x\right)+t_{\alpha} \sin \left(t_{\alpha} \log x\right)\right] .
$$

For simplicity, from now on we will consider this last situation; so $M$ will be assumed large enough.

As we mentioned in the Introduction, the properties of the complex or non trivial zeros of the Riemann's zeta function has been extensively studied[3]. For example it has been demonstrated that there are infinitely many complex zeros and that all of them lie inside the region $0<\Re(s)<1$ and are symmetric about the real axis $\Im(s)=0$. Also is well known that if $\xi(s):=\frac{s}{2}(s-1) \pi^{-s / 2} \Gamma\left(\frac{s}{2}\right) \zeta(s)$ then the non trivial zeros of $\zeta(s)$ are precisely the zeros of $\xi(s)$ and since $\xi(s)=\xi(1-s)$, we have that the complex zeros of $\zeta(s)$ are symmetric with respect to the so called critical line $\Re(s)=1 / 2$. The Riemann Hypothesis is the statement that all the complex zeros have their real part exactly on the critical line: $\sigma_{\alpha}=1 / 2 \forall \alpha$. Although billions of complex zeros have been numerically calculated[11] confirming all of them this conjecture, the full demonstration of the general validity of the Riemann Hypothesis remains still open.

\section{SUPPORTING THE RIEMANN HYPOTHESIS FROM THE MODEL}

We wish to analyze the behavior of the equilibrium grand partition potential (Eq.7), with $\pi(M)$ given by Eqs.(8),12), as a function of the quantities $\sigma_{\alpha} \in(0,1)(\alpha=1,2, \cdots)$. These numbers must be thought as possible values for the real part of the zeta function zeros and our goal would be to identify the true ones.

We focus into a generic zero $\rho_{\gamma}$ and his complex conjugate $\rho_{\gamma}^{*}$ (which is known is also a zero) and observe that, since Riemann actually didn't know the reliable values of the complex zeros, Eqs. (10) and (12) must be taken as general enough as to contemplate the possibility that, associated to $t_{\gamma}=\Im\left(\rho_{\gamma}\right)$, both cases, $\sigma_{\gamma} \neq 1-\sigma_{\gamma}$ and $\sigma_{\gamma}=1-\sigma_{\gamma}$, can be considered for $\Re\left(\rho_{\gamma}\right)$. 
Explicitly we rewrite Eq.(7) for $\beta, M$ and $\mu$ fixed

$$
\Omega\left(\beta, M, \mu ; \sigma_{\gamma}\right)=-\frac{1}{\beta} \log (1+z)^{\pi\left(M ; \sigma_{\gamma}\right)},
$$

with

$$
\pi\left(M ; \sigma_{\gamma}\right)=\bar{\pi}(M)+\widetilde{\pi}_{\neq \gamma}(M)+\tilde{\pi}_{\gamma}\left(M ; \sigma_{\gamma}\right) .
$$

Here the term $\widetilde{\pi}_{\neq \gamma}(M)$ includes all the complex zeros except those labelled $\gamma$ and we assume that its contribution to the prime-counting function is the true one. We write the remaining term:

$\tilde{\pi}_{\gamma}\left(M ; \sigma_{\gamma}\right) \approx \begin{cases}-\frac{4}{\log M}\left(\frac{M^{\sigma \gamma}}{\sigma_{\gamma}^{2}+t_{\gamma}^{2}}\left[\sigma_{\gamma} \cos \left(t_{\gamma} \log M\right)+t_{\gamma} \sin \left(t_{\gamma} \log M\right)\right]+\right. & \\ \left.\frac{M^{\left(1-\sigma_{\gamma}\right)}}{\left(1-\sigma_{\gamma}\right)^{2}+t_{\gamma}^{2}}\left[\left(1-\sigma_{\gamma}\right) \cos \left(t_{\gamma} \log M\right)+t_{\gamma} \sin \left(t_{\gamma} \log M\right)\right]\right) & \text { for } \sigma_{\gamma} \neq 1-\sigma_{\gamma} \\ -\frac{4}{\log M} \frac{M^{1 / 2}}{\frac{1}{4}+t_{\gamma}^{2}}\left[\frac{1}{2} \cos \left(t_{\gamma} \log M\right)+t_{\gamma} \sin \left(t_{\gamma} \log M\right)\right] & \text { for } \sigma_{\gamma}=1-\sigma_{\gamma},\end{cases}$

where we have taken into account the fact that if $\zeta\left(\sigma_{\gamma}+i t_{\gamma}\right)=0$ then also $\zeta\left(1-\sigma_{\gamma}+i t_{\gamma}\right)=$ $0, \zeta\left(\sigma_{\gamma}-i t_{\gamma}\right)=0$ and $\zeta\left(1-\sigma_{\gamma}-i t_{\gamma}\right)=0$.

Special cases can make the analysis even simpler. For $M=\left[M_{1}\right]([\bullet]$ integer part of $\bullet$ ) with $M_{1}=\exp \left[\frac{\pi}{2}(4 n-1) /\left|t_{\gamma}\right|\right]$ and $M=\left[M_{2}\right]$ with $M_{2}=\exp \left[\frac{\pi}{2}(4 n-3) /\left|t_{\gamma}\right|\right](n \in \mathbb{N}$ large enough) and taking into account that $\left|t_{\alpha}\right|>14.1347$ for all the non trivial zeros of the zeta function[3], we have

$$
\tilde{\pi}_{\gamma}\left(M ; \sigma_{\gamma}\right) \approx\left\{\begin{array}{lr} 
\pm \frac{4}{\left|t_{\gamma}\right| \log M_{1,2}}\left(M_{1,2}^{\sigma_{\gamma}}+M_{1,2}^{\left(1-\sigma_{\gamma}\right)}\right) & \text { for } \sigma_{\gamma} \neq 1-\sigma_{\gamma} \\
\pm \frac{4}{\left|t_{\gamma}\right| \log M_{1,2}} M_{1,2}^{1 / 2} & \text { for } \sigma_{\gamma}=1-\sigma_{\gamma},
\end{array}\right.
$$

where the upper sign corresponds to $M_{1}$. Note the avoidable discontinuity in the function $\tilde{\pi}_{\gamma}\left(M ; \sigma_{\gamma}\right)$ when $\sigma_{\gamma}=1-\sigma_{\gamma}$.

Using Eq.(13) together with Eq.(15) -or the particular cases given by Eq.(16)- is easy to see that the grand potential has a unique extremum (minimum or maximum depending on $M)$ at the interval $0<\sigma_{\gamma}<1$. From a heuristic point of view it is reasonable to expect that this extremum (maximum = unstable equilibrium; minimum $=$ stable equilibrium) 
be reached when $\sigma_{\gamma}$ takes the value $\left(\sigma_{\gamma}\right)_{\text {true }}$ that gives, through Eq.(14), the correct timeinvariant number of prime numbers lying inside the interval $[1, M]$.

The same conclusion can be achieved more formally by considering the problem from a dynamic point of view. To this, we firstly take into account that fixing a given value for $\sigma_{\gamma}$ can be thought as imposing a constraint that limits (through $\pi\left(M ; \sigma_{\gamma}\right)$ ) the number of the accessible states to the system. If this constraint is removed, so $\sigma_{\gamma}$ is leaved to freely vary in the interval $(0,1)$, then the equilibrium probability $P\left(\sigma_{\gamma}\right)$ that the system be in states with the parameter taking values in the interval between $\sigma_{\gamma}$ and $\sigma_{\gamma}+\delta \sigma_{\gamma}$ behaves as (see Appendix C)

$$
P\left(\sigma_{\gamma}\right) \propto\left\{\begin{array}{cc}
\exp \left[-\beta \Omega\left(\beta, M, \mu ; \sigma_{\gamma}\right)\right] & \text { if } \sigma_{\gamma} \in(0,1) \\
0 & \text { if } \sigma_{\gamma} \notin(0,1) .
\end{array}\right.
$$

We can then arbitrarily introduce a second random variable such that (17) be the marginal of the joint probability of both ones. We use as new variable a Maxwellian one $\dot{\sigma}_{\gamma}$ so that the bi-dimensional random variable $\left(\sigma_{\gamma}, \dot{\sigma_{\gamma}}\right)$ describes the phase space of an hypothetical particle of mass $m$ moving in a force field with potential function given by

$$
U\left(\sigma_{\gamma}\right)=\left\{\begin{array}{cc}
\Omega\left(\beta, M, \mu ; \sigma_{\gamma}\right) & \text { if } \sigma_{\gamma} \in(0,1) \\
\infty & \text { if } \sigma_{\gamma} \notin(0,1) .
\end{array}\right.
$$

In this picture, $P\left(\sigma_{\gamma}\right)$ can be thought as the marginal of the joint probability distribution

$$
P\left(\sigma_{\gamma}, \dot{\sigma_{\gamma}}\right)=\frac{1}{Z(\beta)} \exp \left\{-\beta\left[U\left(\sigma_{\gamma}\right)+\frac{1}{2} m{\dot{\sigma_{\gamma}}}^{2}\right]\right\} .
$$

Here the normalization constant $Z(\beta)$ is the single particle canonical partition function

$$
Z(\beta)=\iint e^{-\beta \mathcal{H}\left(\sigma_{\gamma}, \dot{\sigma}_{\gamma}\right)} d \sigma_{\gamma} d \dot{\sigma_{\gamma}}
$$

where $\mathcal{H}\left(\sigma_{\gamma}, \dot{\sigma}_{\gamma}\right)$ denotes the Hamiltonian

$$
\mathcal{H}\left(\sigma_{\gamma}, \dot{\sigma_{\gamma}}\right)=\frac{1}{2} m{\dot{\sigma_{\gamma}}}^{2}+U\left(\sigma_{\gamma}\right)
$$

The Hamilton equations of motion for the variables $\sigma_{\gamma}$ and $\dot{\sigma}_{\gamma}$ are

$$
\dot{\sigma_{\gamma}}=\frac{\partial \mathcal{H}}{m \partial \dot{\sigma}_{\gamma}}, \quad m \ddot{\sigma_{\gamma}}=-\frac{\partial \mathcal{H}}{\partial \sigma_{\gamma}},
$$

which yield the Newton equation 


$$
m \frac{d \sigma_{\gamma}}{d t}=-\frac{d U\left(\sigma_{\gamma}\right)}{d \sigma_{\gamma}}
$$

If we assume that initially the particle is placed at the position $\sigma_{\gamma_{0}}=\left(\sigma_{\gamma}\right)_{\text {extr }}$ that extremizes $\Omega\left(\beta, M, \mu ; \sigma_{\gamma}\right)$ with velocity $\dot{\sigma}_{\gamma_{0}}=0$, then the equation of motion has as the unique obvious solution the isolated fixed point $\sigma_{\gamma}(t)=\left(\sigma_{\gamma}\right)_{\text {extr }} \forall t$. Taking into account that for any other pair of initial conditions the solution is time-dependent and that in our lattice gas model the number $\pi\left(M ; \sigma_{\gamma}\right)$ of prime numbers less or equal than $M$ does not

change with time but remains constant, we deduce that it must be $\left(\sigma_{\gamma}\right)_{\text {true }} \equiv\left(\sigma_{\gamma}\right)_{\text {extr }}$. One then infers that $\left(\sigma_{\gamma}\right)_{\text {true }}$ should verify

$$
\left.\frac{\partial \Omega\left(\beta, M, \mu ; \sigma_{\gamma}\right)}{\partial \sigma_{\gamma}}\right|_{\sigma_{\gamma}=\left(\sigma_{\gamma}\right)_{\text {true }}}=-\left.\frac{1}{\beta} \log (1+z) \frac{\partial \widetilde{\pi}_{\gamma}\left(M ; \sigma_{\gamma}\right)}{\partial \sigma_{\gamma}}\right|_{\sigma_{\gamma}=\left(\sigma_{\gamma}\right)_{\text {true }}}=0 .
$$

Derivation of expressions (15) or (16) shows that this equation has the form $\left[f\left(\sigma_{\gamma}\right)-f\left(1-\sigma_{\gamma}\right)\right]_{\sigma_{\gamma}=\left(\sigma_{\gamma}\right)_{\text {true }}}=0$ (with $\left.f\left(\sigma_{\gamma}\right) \neq 0 ; f\left(1-\sigma_{\gamma}\right) \neq 0\right)$ which is clearly verified by $\left(\sigma_{\gamma}\right)_{\text {true }}=1-\left(\sigma_{\gamma}\right)_{\text {true }}=1 / 2$. Note that the maximum (minimum) achieved at $1 / 2$ when the discontinuity is avoided is smaller (larger) than the corresponding values taken by the function $\tilde{\pi}_{\gamma}\left(M ; \sigma_{\gamma}\right)$ in (15) or (16) when $\sigma_{\gamma}=1-\sigma_{\gamma}$.

Because the zero $\rho_{\gamma}$ that we have considered is a generic one, we would conclude that $\left(\sigma_{\alpha}\right)_{\text {true }}=1 / 2 \forall \alpha$, say that, in fact, the real part of all non-trivial zeros of Riemann's zeta function lie on the critical line.

\section{Acknowledgements}

I thank very much Dr. Osvaldo H. Scalise for very useful discussions during the preparation of the manuscript. Support of this work by Consejo Nacional de Investigaciones Científicas y Técnicas, Universidad Nacional de La Plata and Agencia Nacional de Promoción Científica y Tecnológica of Argentina is greatly appreciated. The author is a member of CONICET. 
APPENDIX A: Variational principle in the grand canonical ensemble

Here we follow Georgii [10] to proof the result given by Eq.(5). For any arbitrary measure $\nu \in \mathcal{M}$, we have, using Jensen inequality together with the definition given by Eq.(44):

$$
\begin{aligned}
\Omega[\nu] & =-\frac{1}{\beta} \sum_{\omega \in \Lambda} \nu(\omega)[-\log \nu(\omega)-\beta u(\omega)+\beta \mu N] \\
& =-\frac{1}{\beta} \sum_{\omega \in \Lambda} \nu(\omega) \log \frac{e^{-\beta[u(\omega)-\mu N]}}{\nu(\omega)} \\
& \geq-\frac{1}{\beta} \log \sum_{\omega \in \Lambda} \nu(\omega) \frac{e^{-\beta[u(\omega)-\mu N]}}{\nu(\omega)}
\end{aligned}
$$

or, taking into account Eq. (3),

$$
\Omega[\nu] \geq-\frac{1}{\beta} \log \Xi=\Omega\left[\nu_{0}\right] .
$$

The equality holds if and only if the random variable $\omega \mapsto \frac{e^{-\beta[u(\omega)-\mu N]}}{\nu(\omega)}$ is constant, i.e., if $\nu=\nu_{0}$ since, in this case, according to Eq.(33), is $\frac{e^{-\beta[u(\omega)-\mu N]}}{\nu(\omega)}=\Xi=$ constant.

APPENDIX B: Equation (12)

For $x$ large enough, is $[3] \operatorname{Li}\left(x^{\rho_{\alpha}}\right) \approx x^{\rho_{\alpha}} / \log x^{\rho_{\alpha}}$ and the dominant term in Eq.(10) is that corresponding to $n=1$ :

$$
\begin{aligned}
\widetilde{\pi}(x) & \approx-2 \operatorname{Re} \sum_{\alpha=1}^{\infty} \operatorname{Li}\left(x^{\sigma_{\alpha}+i t_{\alpha}}\right) \\
& \approx-2 \operatorname{Re} \sum_{\alpha=1}^{\infty} \frac{x^{\sigma_{\alpha}+i t_{\alpha}}}{\log x^{\sigma_{\alpha}+i t_{\alpha}}} .
\end{aligned}
$$

Using $x^{i t_{\alpha}}=e^{i t_{\alpha} \log x}$ we have

$$
\begin{aligned}
\tilde{\pi}(x) & \approx-\frac{2}{\log x} \operatorname{Re} \sum_{\alpha=1}^{\infty} \frac{x^{\sigma_{\alpha}}\left[\cos \left(t_{\alpha} \log x\right)+i \sin \left(t_{\alpha} \log x\right)\right]}{\left(\sigma_{\alpha}+i t_{\alpha}\right)} \\
& \approx-\frac{2}{\log x} \operatorname{Re} \sum_{\alpha=1}^{\infty} \frac{x^{\sigma_{\alpha}}\left[\cos \left(t_{\alpha} \log x\right)+i \sin \left(t_{\alpha} \log x\right)\right]\left(\sigma_{\alpha}-i t_{\alpha}\right)}{\sigma_{\alpha}^{2}+t_{\alpha}^{2}}
\end{aligned}
$$

from where Eq.(12) follows. 


\section{APPENDIX C: The Probability $P\left(\sigma_{\gamma}\right)$}

In this appendix we deduce Eq. (17) for $P\left(\sigma_{\gamma}\right)$ by applying to our case the method of Reif[12]. We call $A_{t}=A \cup A_{r}$ the total isolated system made up of the lattice gas and the reservoir at temperature $T_{r}$ and chemical potential $\mu_{r}$. In equilibrium the temperature and chemical potential of $A$ equals those of $A_{r}: T=T_{r} ; \mu=\mu_{r}$. We are assuming that $A$ (and consequently $\left.A_{t}\right)$ depends on $\sigma_{\gamma}$ through $\pi\left(M ; \sigma_{\gamma}\right)$ so that if $\sigma_{\gamma}$ changes from a given value $\sigma_{\gamma}=\sigma_{\gamma 1}$ to a generic one $\sigma_{\gamma}$, then the thermodynamical functions of $A$ (i.e. $H, \nu(u), \nu(N)$ or $\Omega$ ) will also change (for example $\Delta H=H\left(\sigma_{\gamma}\right)-H\left(\sigma_{\gamma 1}\right)$ ) and so do the corresponding thermodynamical functions of $A_{t}$ (e.g. $\left.\Delta H_{t}=H_{t}\left(\sigma_{\gamma}\right)-H_{t}\left(\sigma_{\gamma 1}\right)\right)$.

Because of the additivity of the entropy, we can write its variation in the total system as $\Delta H_{t}=\Delta H+\Delta H_{r}$. But the variation in the reservoir entropy is given by $\Delta H_{r}=-\beta Q$ where $Q$ denotes the heat absorbed by $A$ from $A_{r}$ and $\beta=\left(k_{B} T\right)^{-1}=\left(k_{B} T_{r}\right)^{-1}$. According to the first law of thermodynamics applied to $A$, the heat $Q$ can be written

$$
Q=\Delta \nu(u)-\mu \Delta \nu(N)+W
$$

where: $\Delta \nu(u)$ is the variation in the internal energy of $A, \Delta \nu(N)$ the variation in the mean particles number of $A$ and $W$ the work done by $A$ along the process.

Then we have

$$
\begin{aligned}
\Delta H_{t}=\Delta H-\beta Q & =\beta\left[\frac{\Delta H}{\beta}-(\Delta \nu(u)-\mu \Delta \nu(N)+W)\right]= \\
& -\beta\left[\Delta\left(\nu(u)-\frac{H}{\beta}-\mu \nu(N)\right)+W\right],
\end{aligned}
$$

or, remembering the definition given by Eq. (41) in the text:

$$
\Delta H_{t}=-\beta[\Delta \Omega+W]
$$

Assuming that the external parameters of $A$ are fixed we have $W=0$, so that the total entropy will change

$$
\Delta H_{t}=H_{t}\left(\sigma_{\gamma}\right)-H_{t}\left(\sigma_{\gamma 1}\right)=-\beta\left[\Omega\left(\sigma_{\gamma}\right)-\Omega\left(\sigma_{\gamma 1}\right)\right]
$$

Now, taking into account that the total system is isolated so that all the micro-states (configurations $\omega$ ) which are accessible to $A_{t}$ have the same probability, we have[12] 


$$
P\left(\sigma_{\gamma}\right) \propto \mathcal{N}_{t}\left(\sigma_{\gamma}\right)=e^{H_{t}\left(\sigma_{\gamma}\right)}
$$

where $\mathcal{N}_{t}\left(\sigma_{\gamma}\right)$ denotes the number of micro-states accessible to $A_{t}$ when the parameter takes values between $\sigma_{\gamma}$ and $\sigma_{\gamma}+\delta \sigma_{\gamma}$. It is worth mentioning that if the equilibrium state of $A_{t}$ when the parameter is fixed at $\sigma_{\gamma}$ is $\nu_{t}\left(\sigma_{\gamma}\right)=\left(\nu_{t}\left(\omega ; \sigma_{\gamma}\right) \mid \omega \in \Lambda_{t}\left(\sigma_{\gamma}\right)\right)$ with $\nu_{t}\left(\omega ; \sigma_{\gamma}\right)=1 / \mathcal{N}_{t}\left(\sigma_{\gamma}\right)$ where $\mathcal{N}_{t}\left(\sigma_{\gamma}\right)=\left|\Lambda_{t}\left(\sigma_{\gamma}\right)\right|$ and $\Lambda_{t}\left(\sigma_{\gamma}\right)$ the configuration space, then the entropy is $H_{t}\left(\sigma_{\gamma}\right)=-\sum_{\omega \in \Lambda_{t}\left(\sigma_{\gamma}\right)} \nu_{t}\left(\omega ; \sigma_{\gamma}\right) \log \nu_{t}\left(\omega ; \sigma_{\gamma}\right)=\log \mathcal{N}_{t}\left(\sigma_{\gamma}\right)$ from where the equality of Eq.(C4) follows. Replacing $H_{t}\left(\sigma_{\gamma}\right)$ in Eq.(C4) by the expression obtained from Eq.(C3) and including into the proportionality constant all the terms which are functions of the arbitrary value $\sigma_{\gamma 1}$ we have $P\left(\sigma_{\gamma}\right) \propto e^{-\beta \Omega\left(\sigma_{\gamma}\right)}$, say Eq.(17) in the text.

[1] J.M. Luck, P. Moussa, M. Waldschmidt (Eds.), Number Theory and Physics, Springer, Berlin, 1990.

[2] M. Waldschmidt, P. Moussa, J.M. Luck, C. Itzykson (Eds.), From Number Theory to Physics, Springer, Berlin, 1992.

[3] H.M. Edwards, Riemann's Zeta Function, Academic Press, New York, 1974.

[4] M.F. Shlesinger, Physica A 138, 310 (1986).

[5] M. Wolf, Physica A 274, 149 (1999).

[6] A. Knauf, Comm. Math. Phys. 153, 77 (1993); 196, 703 (1998).

[7] B. Julia, Statistical theory of numbers, in: J.M. Luck, P. Moussa, M. Waldschmidt (Eds.), Number Theory and Physics, Springer, Berlin, 1990, p.276.

[8] D. Schumayer, D.A.W. Hutchinson, Rev. Mod. Phys. 83, 307 (2011).

[9] C.P. Willans, Mathematical Gazette 48, 413 (1964).

[10] H-O Georgii, Gibbs Measures and Phase Transitions ( Walter de Gruyter, Berlin, 1988).

[11] A.M. Odlyzko, http://www.dtc.umn.edu/odlyzko/

[12] F. Reif, Fundamentals of Statistical and Thermal Physics, McGraw-Hill, New York, 1965. 